\title{
8
}

\section{Video Processing Techniques for Assisted CCTV Inspection and Condition Rating of Sewers}

\author{
Nima Sarshar, Mahmoud R. Halfawy and \\ Jantira Hengmeechai
}

This chapter describes the development of a software system to semiautomatically extract historical condition data information from archived sewer inspection CCTV files. The software represents and manages the sewer data in a centralized repository. Users can easily access inspection and condition data via a GIS interface. The software implements video processing algorithms to support semi-automatic evaluation of the CCTV recording by automatically detecting defect features and assisting the user to define scores and rating according to a pre-defined condition rating scheme. The software was successfully used to efficiently access, analyze, and evaluate sewer condition data from CCTV video files obtained from the City of Regina.

\subsection{Introduction}

Sewer inspection technologies have rapidly advanced over the last two decades. Methods such as closed-circuit television (CCTV), sewer scanner and evaluation technology (SSET), focused electrode leak location (FELL), ultrasonic inspection, laser-based scanning, and ground penetrating radar

Sarshar, N., M. Halfawy and J. Hengmeechai. 2009. "Video Processing Techniques for Assisted CCTV Inspection and Condition Rating of Sewers." Journal of Water Management Modeling R235-08. doi: 10.14796/JWMM.R235-08.

(C) CHI 2009 www.chijournal.org ISSN: 2292-6062 (Formerly in Conceptual Modeling of Urban Water Systems. ISBN: 978-0-9808853-2-3) 
(GPR) have been in use in many parts of the world. However, CCTV remains, by far, the most widely used technology for inspecting the internal surface of non man-entry sewers. For over 40 years, municipalities, especially in North America, have been using CCTV as the primary sewer inspection method (Halfawy et al. 2000).

The CCTV technique involves the use of a robot-mounted forwardlooking pan/tilt and zoom camera that travels between two manholes. CCTV equipment is operated by certified operators who are trained to effectively control the camera and interpret video streams to deduce an accurate description of the structural condition of the sewer. CCTV can identify defects such as longitudinal/circumferential cracks, fractures, deformation, collapse, breaks, open or displaced joints, surface abrasion or corrosion, tree root penetration, encrustation, and lateral connections. Each sewer is rated based on the number and severity of defects. Visual inspection data are coded according to a standard coding system. The defect codes are then assigned scores based on their severity. These scores are then used to calculate the total, peak, and mean scores. Based on these scores, a condition grade is calculated on a one-five scale. A sewer condition rating is primarily used for estimating remaining service life and the probability of failure, and to guide inspection and renewal planning decisions (Halfawy et al. 2008).

Sewer historical condition information is critical for establishing deterioration patterns, and hence, the development of statistical models to predict future condition of the sewers. Such information is the key enabler for developing proactive asset management and renewal planning strategies. Lack of adequate historical condition data and reliable deterioration models have caused asset management and renewal planning decisions to be based, in the most part, on "educated guesses" and "subjective prediction" of sewer future condition (Halfawy 2008). Most municipalities around the world possess thousands of hours of archived CCTV video recordings and inspection reports, which could provide historical information about the condition of the asset and to objectively predict the state of the sewers in future. While millions of dollars have been spent on gathering these CCTV recordings, unfortunately, they cannot be feasibly organized and analyzed to extract meaningful condition data.

A number of issues contribute to this problem. First, many of the paperbased inspection reports may have been lost over the years or cannot be easily referenced to a particular video footage. Second, many of the recordings may have been inspected and rated using different defect coding schemes, which would create inconsistency in the data recorded in the 
inspection reports, which would require mapping between these different schemes.

There has been a number of recent works dealing with automated detection of defects in sewer pipelines. Most of these works propose the use of new techniques, e.g. Gooch, 1996. The use of image processing techniques for automated identification of cracks in sewer pipes has been studied by several researchers, e.g. Sinha and Knight (2004), Iyer and Sinha (2005), and Iyer and Sinha (2006). The work reported in this chapter is an attempt to automate the detection of other types of defects, e.g. excessive debris, penetrating roots, and displaced joints, directly from CCTV files.

Analyzing, re-evaluating and organizing archived CCTV video recordings could assist municipalities in retrieving historical condition data of the sewers. Clearly, re-organization and re-evaluation of these recordings in a manual fashion is technically and economically infeasible. This chapter presents an attempt to develop a software solution that would enable the use of archived sewer inspection CCTV files and the implementation of advanced video analysis techniques to semi-automatically extract the muchneeded historical condition data in a cost-effective manner.

\subsection{Service-Oriented Architecture for Assisted Sewer Inspection and Condition Rating}

A service-oriented architecture (SOA) for assisted CCTV-based sewer inspection and re-evaluation was developed. Figure 8.1 shows the main components and interfaces of the proposed architecture. The proposed architecture relies on a GIS-based interface for integrating the sewer inspection and condition assessment data into an asset management data repository (Halfawy and Figueroa 2006, Halfawy et al. 2002) that facilitates efficient data access and decision-making. The intelligent video processing (IVP) components are responsible for re-inspecting archived video recordings and populating the sewer data repository with standard, uniform, historic condition data extracted from archived CCTV recordings.

A prototype software system was developed based on the proposed architecture. A typical operation cycle of the system can be briefly described as follows:

1. The user selects a set of sewers for re-evaluating their CCTV data.

2. The system looks up the repository to see if archived recordings for any of these sewers are available. 
3. The GIS then invokes the intelligent video processing (IVP) services to generate condition data for these sewers, through re-evaluating corresponding CCTV recordings.

4. The IVP exports the evaluation job to a network of distributed video processors that might be distributed over many different machines. The user will be notified when the processing is complete.

5. The IVP generates an interim inspection report (IIR) for each video footage.

6. After receiving an IIR, the user can inspect the report, override some decisions made by the IVP on defect types and severity, or add comments to the report.

7. The IIR is then passed to a condition rating web service to transform coded inspection data to scoring and condition grades.

8. The sewer inspection data repository will be updated with the new inspection data and condition rating indices, and the user can view them on the GIS interface.

The rest of this chapter describes the main implementation steps of the proposed architecture. The functionality of various components is demonstrated using the CCTV data sets obtained from the City of Regina.

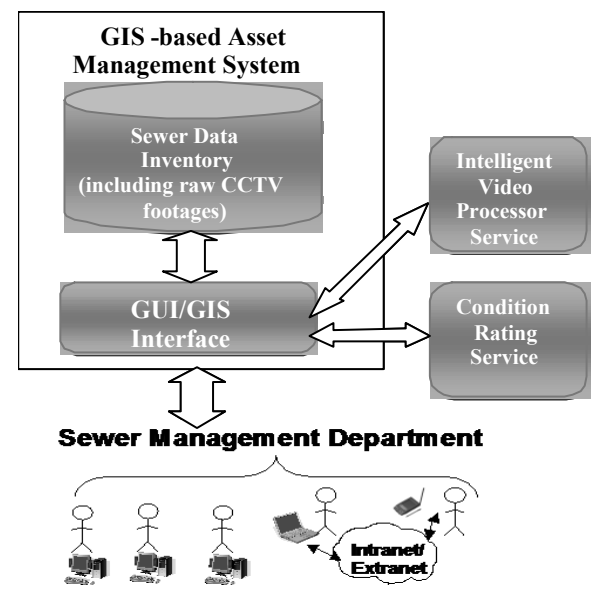

Figure 8.1 Service-oriented architecture for sewer inspection and condition assessment software. 


\subsection{Implementation of the GIS Interface and Data Management Component/Service}

This component provides a GIS interface and data management services to enable efficient storage, access, analysis, and management of sewer inspection and condition data. The component was implemented as an addon to the ArcGIS software using the ESRI ArcObjects class library. An object-oriented unified modeling language (UML) data model that integrates spatial and non-spatial data for sanitary and storm sewers was developed (Halfawy and Figueroa 2006). Based on the ESRI water utilities spatial data models, the model represented a comprehensive view of the sewer asset data including structural, condition, and performance characteristics. The data model was input into the ArcCatalog's Schema Wizard, which generated the database tables, fields, and data types based on the ESRI geodatabase objectrelational schema.

The City of Regina uses the flexidata software (www.flexi-data.com/) for recording inspection data, defect scoring, and calculation of the WRC sewer condition rating (WRc 2001). The CCTV data are exported from the software and used to populate relevant attributes in the sewer data repository. These attributes included the defects and their scoring across the sewer centerline, inspection date, tape number, start/finish manholes, direction, etc. The software enables efficient access to the data repository to retrieve the latest and historical CCTV inspection data and condition ratings. Figure 8.2 shows a typical screenshot of the inspection and condition assessment interface.

The component implements a number of data management functions to query the sewer network based on the sewer material, diameter, age, inspection records, or condition state. Summary charts for the entire network showing the total length or number of sewers within various categories can also be displayed. The component can be used by CCTV operators and City engineers to access and manage CCTV data. CCTV operators can use the software inside the CCTV truck instead of carrying paper maps in the field. An operator can select a sewer on the map, access detailed data about the sewer and its inspection history. CCTV data can be directly entered using standard forms. The data can be easily retrieved later in the office from the GIS map. Video files can be played and graphical presentation of the inspection data can be generated.

Query of condition data can also be run and displayed. Color-coded maps of the sewer network condition can be easily generated. These maps could 
help identify and prioritize sewers for maintenance and rehabilitation actions. Figure 8.3 shows a typical map in which sanitary sewers with condition rating of " 3 " would be shown in contrasting colour (not visible in gray scale printing)

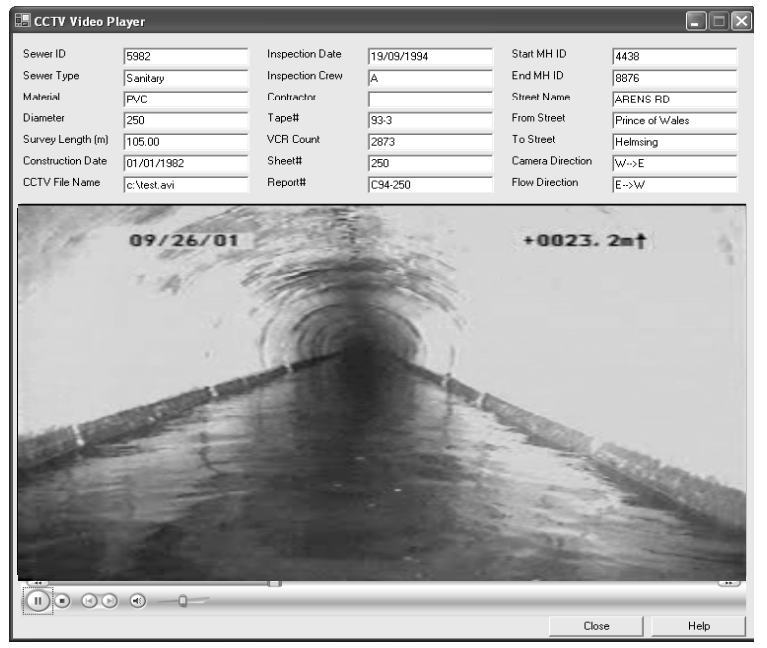

Figure 8.2 CCTV inspection and condition assessment interface.

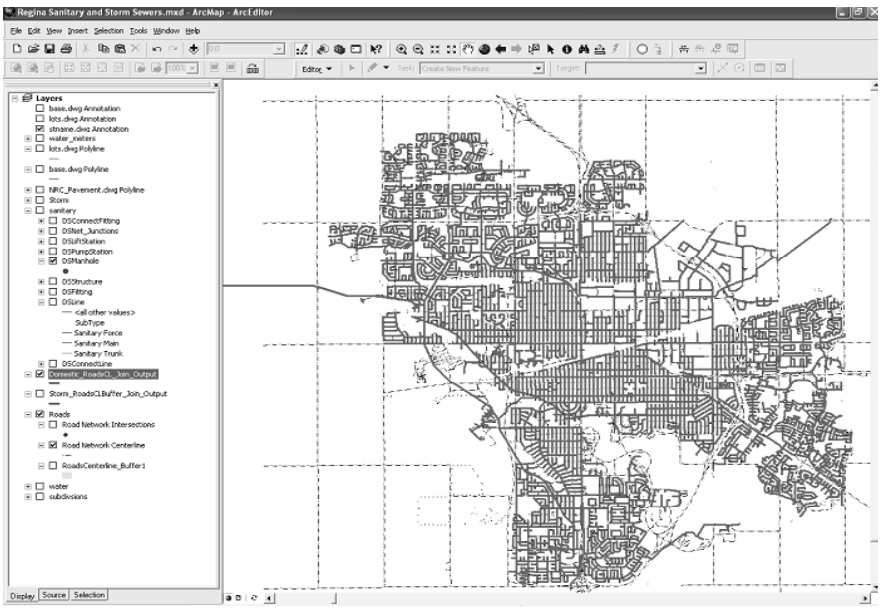

Figure 8.3 GIS representation of query result to identify sanitary sewers with condition rating " 3 ." (not visible in gray scale) 


\subsection{Implementation of the Video Processing Component/Service}

The IVP is a distributed network of three-tier web services architecture that implement video processing algorithms (Figure 8.4). The tiered architecture ensures the scalability of the system as it grows into potentially hundreds of users and processing machines. The three tiers are:

1. Tier 1 consists of the clients that connect to the IVP for reevaluating CCTV recordings. This may include different municipalities or other customers, usually through their GIS-based interfaces.

2. Tier 2 is the system middleware, which manages the interactions with client applications. This tier is responsible for queuing the jobs, breaking down the large inspection jobs into smaller pieces and distributing them between different processors, balancing the load and managing data integrity.

3. Tier 3 hosts the actual components that implement the video processing algorithms. Video processing services in this tier are exposed to the middleware through a standardized web service interface.

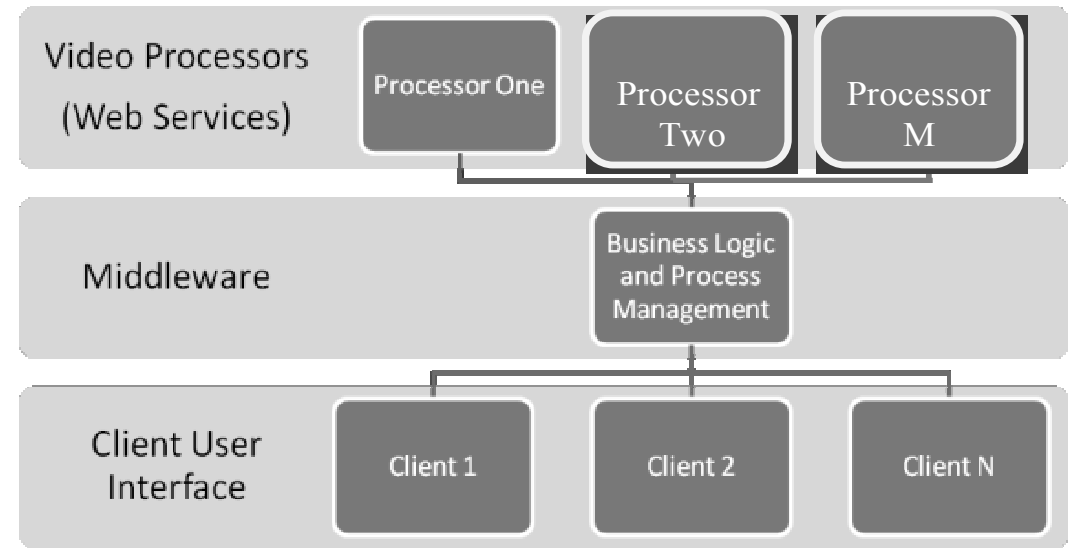

Figure 8.4 The 3-tier architecture of the IVP services. 


\subsection{Video Processing Algorithms}

This section describes the video processing algorithms implemented in the IVP web service to extract sewer condition data from CCTV video recordings.

\subsubsection{Camera motion analysis for identifying "areas of interest"}

In a typical CCTV video footage, the operator's movement of the camera can provide important clues about the areas of interest that the operator encounters during the inspection session. By tracking and analyzing the movement of the camera, these clues can be used to identify "areas of interest" within the video stream, which could be further processed to extract useful information. Generally, when an operator encounters a typical "area of interest" in the sewer, the operator would either (i) stop to take notes, (ii) move the camera back and forth or (iii) tilt/pan or zoom the camera towards the walls or bottom of the sewer to capture a defect more accurately.

An algorithm to analyze the motion of the camera in the sewer to identify "areas of interest" to the original operator was developed. The algorithm primarily identifies the video segments where the operator stops the camera or moves the camera backward. Analyzing the motion of the camera serves two main purposes: (i) by finding video segments for areas of interest, the defect detection task can be limited to only those areas in the video segment that are expected to contain defects, thus significantly reducing the computational time needed and the rate of false positives; (ii) the motion of the camera helps to identify the areas in the video segment that the operator has found to be interesting, and are most likely to contain defects. The algorithm works as follows:

1. Frames are read one by one from the video file. A time series signal $D(t)$ is created in which the component at time " $\mathrm{t}$ " is the normalized mean absolute difference between the video at frame $t$ and $t+1$. This is a noisy time series; even when the camera completely stops, there can be significant differences between the two frames.

2. The moving average $\mathrm{M}(\mathrm{t})$ and standard deviation $\mathrm{V}(\mathrm{t})$ of the time series up until the current time " $t$ " is calculated.

3. At each time " $t$ " if $D(t)>M(t)+r V(t)$, for some constant fixed value of $r$ (default $=0.5$ ) then the frame " $t$ " is flagged "suspicious". Let $\mathrm{S}(\mathrm{t})$ be the time series in which $\mathrm{S}(\mathrm{t})=1$ if frame $t$ is "suspicious," and equals 0 otherwise. The 
"suspicious" frames indicate the frames in which the initial operator might have had an interest. Only "suspicious" frames are further processed for finding possible defects.

4. Once all the frames are processed, video segments of "areas of interest" are produced by processing the series of suspicious frames as follows. Firstly, the time series $S(t)$ is median filtered with a relatively small filter length (default is 30 frames or $1 \mathrm{~s}$ ). The resulting series is median filtered again, this time with a much larger filter length (default=300 frames, or $10 \mathrm{~s}$ ). Each connected segment of non zero values in the resulting time series corresponds to a segment of the video that includes a potential "area of interest."

5. The positions of the "areas of interest" segments are written to the interim report as "Alerts."

\subsubsection{Camera Position Approximation for Locating "Areas of Interest"}

The position of individual frames within "areas of interest," which will be used to identify the type and severity of defects, should be determined accurately. Since the sewer length (manhole to manhole) is usually known from the video file or the GIS database, an algorithm to estimate the position of the camera inside the sewer in any given frame needs to be developed. However, since the camera makes frequent stops in the video and moves back and forth frequently, the video time-line itself can be a poor indication of the relative position of the camera inside the sewer. Instead of using the video time-line, a more robust algorithm to estimate the velocity of the camera and integrate it to estimate the position of the camera was developed.

An initial attempt was made by devising an algorithm that uses the average absolute pixel difference between consecutive frames as an indication of the camera velocity. However, this algorithm yielded inaccurate results due to the fact that it cannot account for the camera movement direction (i.e. forward vs. backward movement). The estimation error increased significantly with the increase of changing the camera moving direction in the video footage.

Therefore, the algorithm was revised to account for the camera moving direction by employing the optical flow technique to estimate the distance of unique features moving in one image frame to another frame. The optical flow technique provided an accurate estimate, due to its ability to account 
for the moving direction in the distance calculation. The forward/backward moving direction is determined by the direction of the optical flow vector. For example, when the camera is moving forward into the sewer, the optical flow vector direction moves outwards from the center of the black hole of the sewer or approximately the center of the images, and vice versa for the backward direction. Figures 8.5 and 8.6 illustrate the directional prediction concept of the optical flow technique.

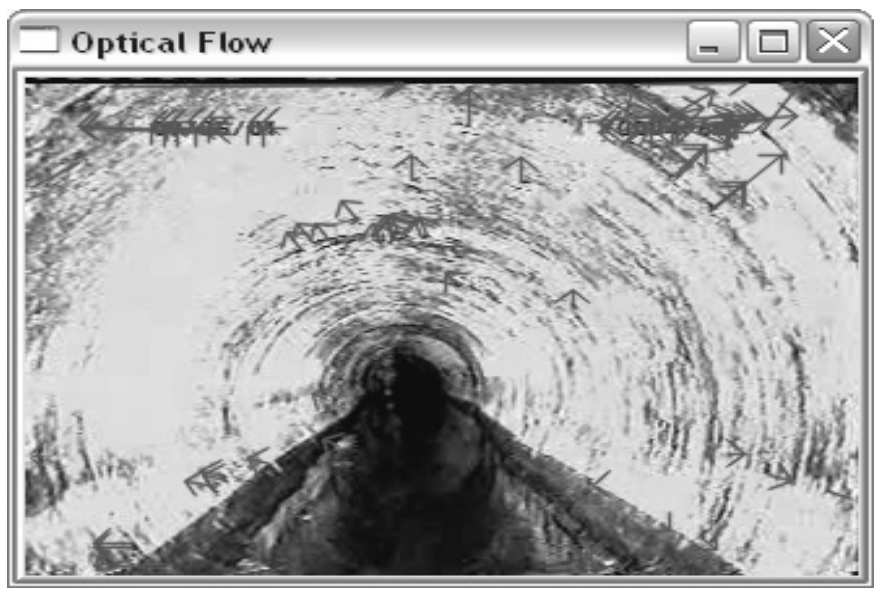

Figure 8.5 Optical flow vectors moving outwards from the center, indicating forward moving direction.

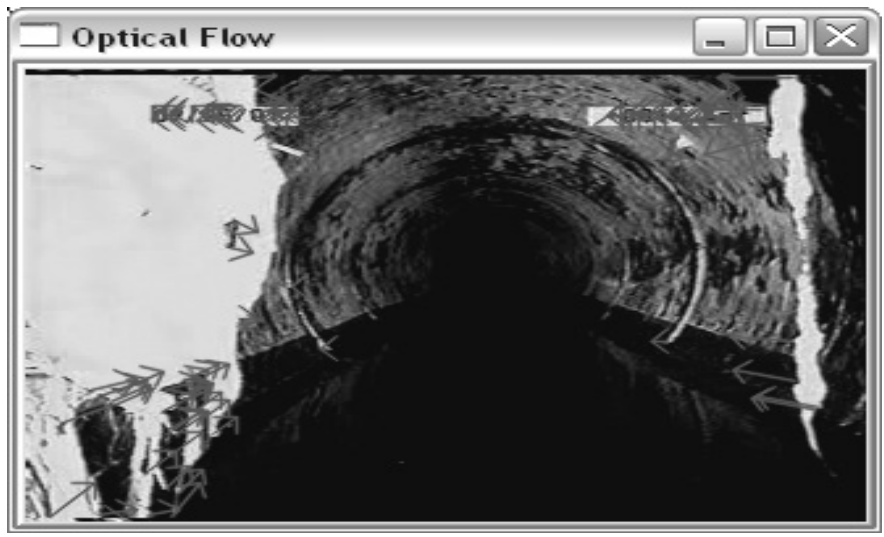

Figure 8.6 Optical flow vectors moving inwards into the center, indicating backward moving direction. 
The proposed algorithm was validated using the absolute frame position data that typically appears on the CCTV footage. Using this data as a reference, the percentage error in estimating the camera position using different approaches was calculated and compared. Comparison of the three methods was conducted by taking thirteen frames from a typical CCTV video file. The calculated distance was then compared with the position displayed on the top right corner of each image. The steps were repeated for the three tested approaches: (i) using the video timeline for reference methods; (ii) estimating the frame position by calculating the pixel difference of consecutive frames; and (iii) using the optical flow technique. The results in Table 8.1 show the percentage of the distance along the sewer centerline.

Table 8.1 Comparison of estimated camera position vs. the actual position for 13 sampled frames from a typical video file using the three approaches.

\begin{tabular}{ccccccc}
\hline $\begin{array}{c}\text { Actual } \\
\text { position } \\
(\%)\end{array}$ & $\begin{array}{c}\text { (1) Video Timeline } \\
\text { Estimated } \\
\text { position (\%) }\end{array}$ & $\begin{array}{c}\text { \% } \\
\text { error }\end{array}$ & $\begin{array}{c}\text { (2) Pixel Difference } \\
\text { Estimated } \\
\text { position (\%) }\end{array}$ & $\begin{array}{c}\text { (3) Optical Flow } \\
\text { \% error }\end{array}$ & $\begin{array}{c}\text { Estimated } \\
\text { position (\%) }\end{array}$ & $\begin{array}{c}\% \\
\text { error }\end{array}$ \\
\hline 17.88 & 12.97 & 27.47 & 15.31 & 14.38 & 17.94 & 0.35 \\
23.77 & 19.45 & 18.18 & 22.91 & 3.64 & 23.71 & 0.28 \\
23.77 & 25.94 & 9.10 & 27.24 & 14.57 & 24.54 & 3.24 \\
24.95 & 32.42 & 29.93 & 32.10 & 28.66 & 26.94 & 7.95 \\
36.35 & 38.90 & 7.03 & 39.25 & 7.99 & 39.12 & 7.64 \\
54.42 & 45.39 & 16.60 & 48.72 & 10.47 & 57.01 & 4.76 \\
58.15 & 51.87 & 10.81 & 55.78 & 4.08 & 61.66 & 6.03 \\
60.31 & 58.35 & 3.25 & 61.73 & 2.34 & 65.54 & 8.66 \\
62.48 & 64.84 & 3.78 & 69.12 & 10.64 & 69.03 & 10.49 \\
85.46 & 71.32 & 16.55 & 77.40 & 9.43 & 83.67 & 2.10 \\
99.02 & 77.80 & 21.42 & 86.05 & 13.09 & 95.46 & 3.59 \\
99.61 & 84.29 & 15.38 & 93.22 & 6.41 & 98.16 & 1.45 \\
100 & 90.77 & 9.23 & 100 & 0 & 100 & 0 \\
\hline Avg. Error & - & 14.52 & - & 9.67 & - & 4.35 \\
\hline
\end{tabular}

This comparison shows that the optical flow method yields the best accuracy for camera position estimating. The average relative error, as shown in Table 8.1 , is less than $4.4 \%$, which is three times more accurate than the time-line itself and two times more accurate than when the absolute difference alone is used. Therefore, the IVP component implemented the 
optical flow method to determine the position of the CCTV camera accurately.

\subsubsection{Video Understanding and Defect Classification}

Once the areas in the video recording that potentially contain defects are found, the next step is to understand and analyze the corresponding frames. The previous sections described the algorithms for identifying the "areas of interest" in the video footage, as well as determining the position of suspicious frames within these areas. Several image-processing algorithms were then implemented to analyze these frames for various types of defects.
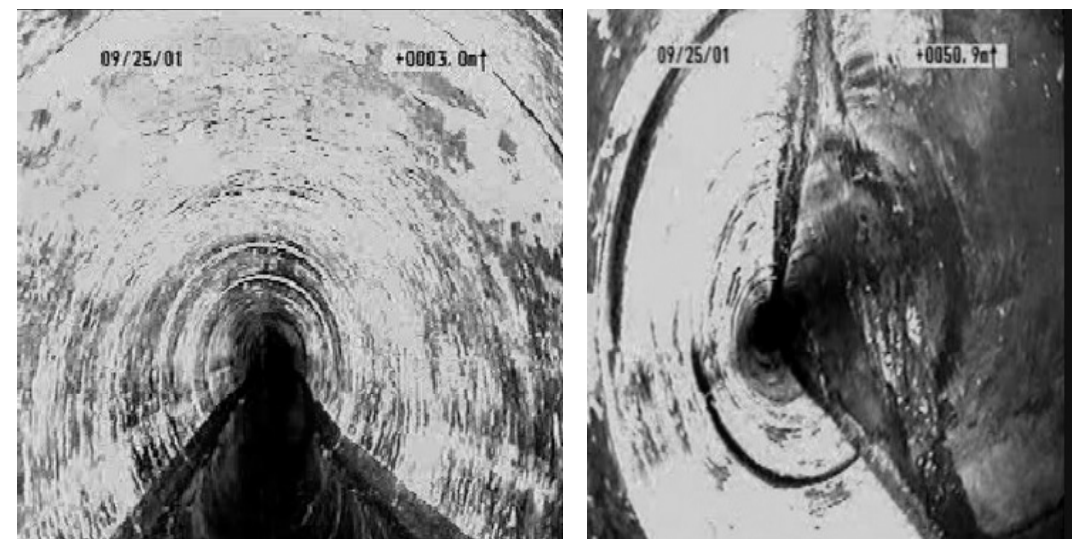

Figure 8.7 An example "Horizontal" (left) and "Vertical" frames (right).

Several defects can generally be identified, including longitudinal or circumferential cracks, fractures, deformation, collapse, open or displaced joints, surface abrasion or corrosion, tree root penetration, encrustation, and lateral connections. Generally, frames of CCTV video files can be divided into two major categories (Figure 8.7):

1. Horizontal (or typical) frames that are captured when the camera is pointing forward towards the end of the sewer. These shots constitute the vast majority of the frames (more than $99.5 \%$ in our samples). Typical images from a sewer are highly structured, consisting of an "end of the tunnel" hole, the walls and the water. 
2. Vertical frames that are captured when the operator spots a suspicious defect on the wall or the bottom of the sewer, or simply wants to capture a shot from the wall for reference purposes.

An algorithm to classify the frames into Horizontal or Vertical was developed. Based on the type of the frame, certain types of defects can be sought. In Horizontal frames, an operator generally looks for cracks and other defects related to the walls (e.g. tree root intrusion). For these frames, a segmentation algorithm was implemented for finding different constituents of the scene, including the end-of-pipe hole, the wall and the water.

\section{Frame Classification to Horizontal or Vertical}

A frame classification technique was implemented to automatically identify whether a video frame contains a significant portion of the wall, compared to the usual camera angle, which is towards the end of the sewer. The classification technique was originally proposed by Viola and Jones (2001) for face detection applications. The IVP component implemented an algorithm that uses Haar Wavelets for feature extraction and AdaBoost for classification. The algorithm works as follows:

1. For each frame in an "area of interest," the algorithm starts by significantly reducing the size of the frame.

2. A median filter with a larger base is used to filter the image. The result is a blurred image in which the end of the tunnel, if it exists, is signified.

3. The algorithm then calculates 200 Haar wavelet features for the resulting image. These Haar features are optimally chosen from a large pool of possible features.

4. These 200 features are then fed into a classifier based on AdaBoost. The classifier has been trained off-line and is capable of detecting the frames that contain a significant portion of the wall.

The training of the classifier is performed offline as follows:

1. Training examples are extracted from the available video files. The file used for training included a total of 300 images that contain a scene from a wall in the pipe, and 300 images that contain the usual end-of-pipe frames. 
2. A total of 10,000 random Haar wavelet features are extracted for each of these training frames.

3. AdaBoost classifier is used to choose a subset of size 200 of these features.

4. The Adaboost classifier is trained using these 200 features to create the final classifier.

The frame classification technique proved to be both efficient and reliable. In all videos that were tested using the IVP component, the positions of all frames that contain a shot of wall were accurately detected.

\section{Segmentation of Typical Frames}

A typical image frame (Figure 8.8, left) can be segmented into three parts: hole, water, and wall segments. Wastes on the water surface and roots on the pipe wall are examples of sewer defects that will stand out after this segmentation. For the hole segment, the location of the hole can be found by analyzing image intensity. The plot of the mean value of grayscale intensity versus position along the $\mathrm{x}$-axis of an image is shown in Figure 8.8. The position at the smallest value would be approximately the center of the hole in the horizontal plane, due to the fact that a darker color area contains less scale value than a lighter color area. The plot of grayscale value at the previously obtained center in $\mathrm{x}$-axis versus the position along the $\mathrm{y}$-axis would yield roughly the top of the hole in the vertical plane, as shown in Figure 8.9. For example, the top of the hole location obtained from the plots in Figure 8.8 and Figure 8.9 is at $(184,175)$ position. Assuming a reasonable value for the radius of the hole (e.g. 15 pixels), the hole segment can be clearly identified. Figure 8.10 shows the segmentation of a typical defect-free frame.

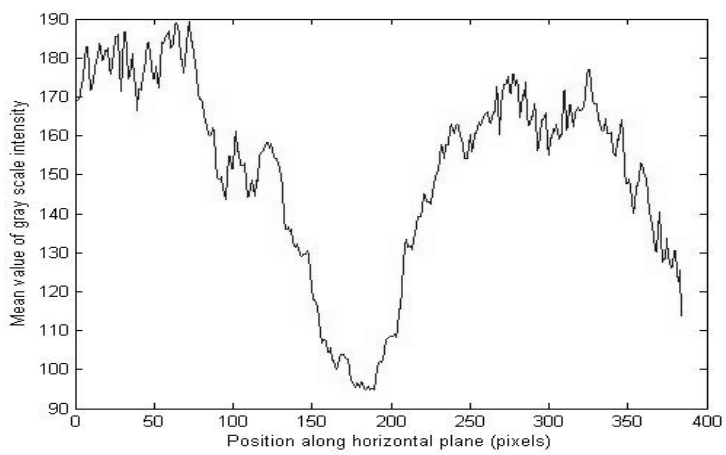

Figure 8.8 The mean value of grayscale intensity along horizontal plane. 


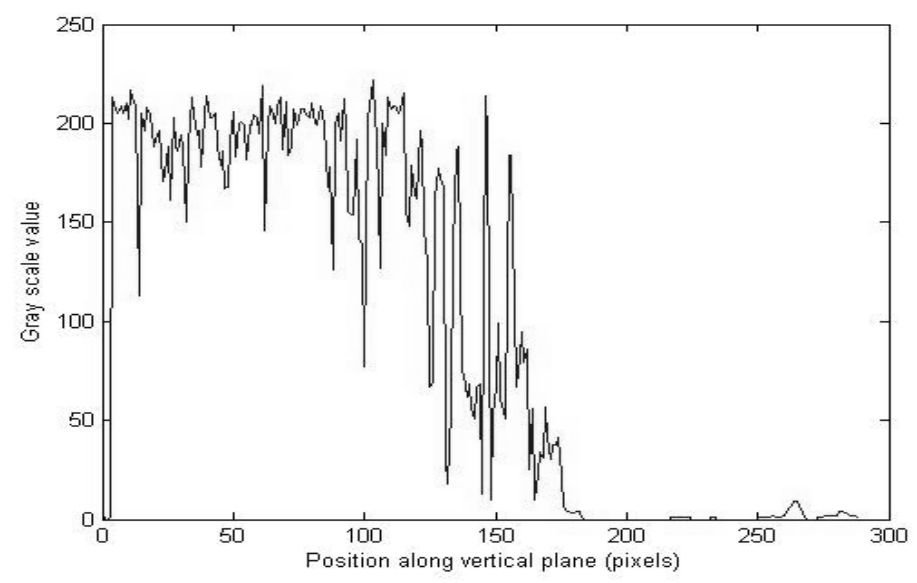

Figure 8.9 The mean value of grayscale intensity at the horizontal center position along vertical plane.

The segmentation of the wall and water bodies was implemented using the image close and erosion techniques in MATLAB, which are implemented using the imclose() and imerode() functions. The imclose() and imerode() functions are used for removing dark spots on the wall, joining the group of black or white color together, and smoothing the outer edges. The original image was first converted to grayscale, and then converted to black and white using a threshold value from graythresh() function, before implementing imclose() and imerode() sequentially. The result from this step was then converted to binary, and was made to have the same number of channels as the original image (RGB).

The black color area is the water segment and the rest is the wall segment. To get the output image with the wall segment, the original image was multiplied by the binary image, where the black color has a value of 0 and the white color has a value of 1 . The water segment is simply obtained by subtracting the original image with the wall segment image.

In defect-free frames, a typical image is segmented into the three distinct segments (Figure 8.10). However, frames that exhibit one or more defects may show some anomalies after segmentation. For example, the water and wall segments in Figure 8.11 show an overlap due to the existence of excessive siltation accumulated at the bottom of the sewer, causing the wall segment to appear as if it were extended into the water segment. This can be detected, and the frame can be marked as containing a potential defect in the water. 


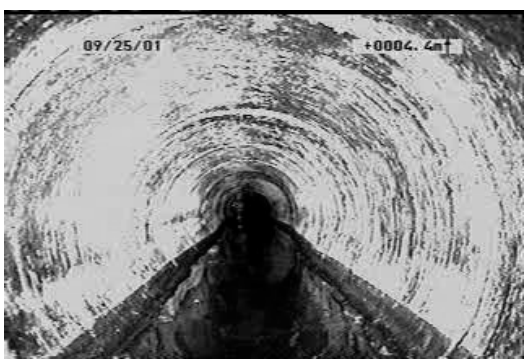

(a) Original image

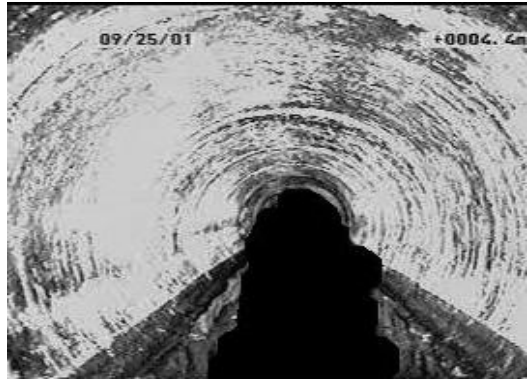

(c) Wall segment

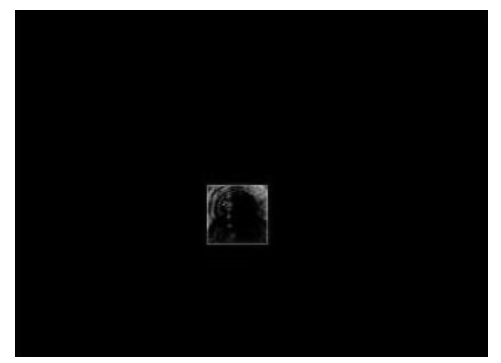

(b) Hole segment

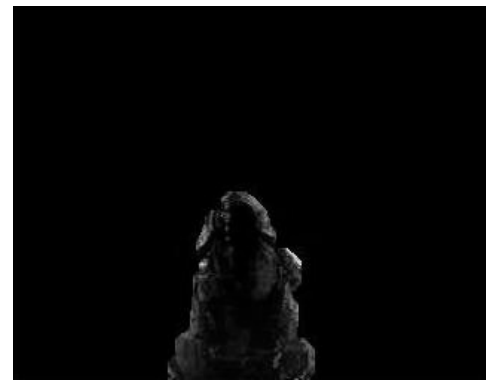

(d) Water segment

Figure 8.10 An example segmentation of a typical defect-free frame.
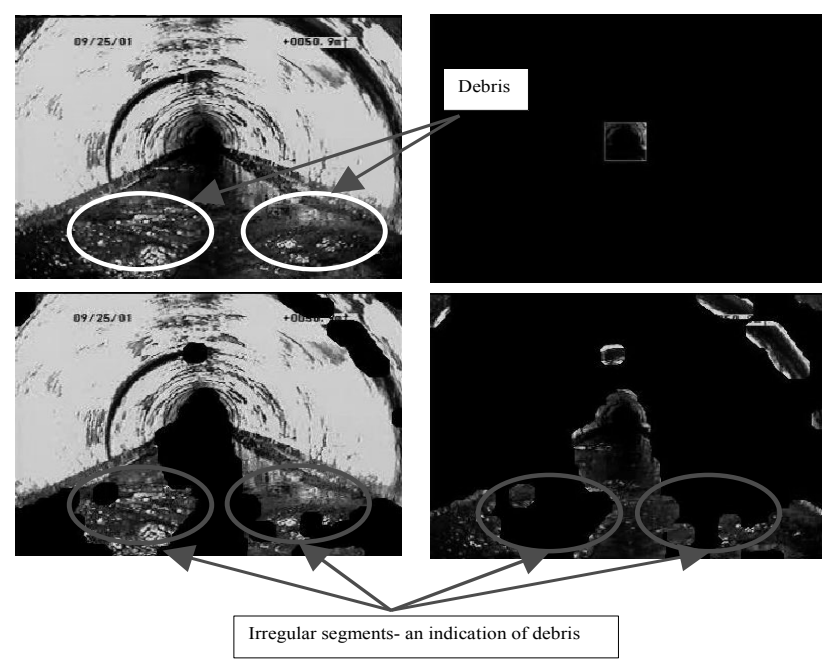

Figure 8.11 An example frame with excessive debris at the water. 


\subsection{Generating and Editing Inspection Reports}

Once the processing of a video recording is completed, an interim inspection report (IIR) is generated in standard XML format that describes the video analysis results. The report defines the position of various "areas of interest" within the CCTV video, the type of defect suggested for each of these areas, and the severity of these defects. Users can edit the IIR through a Graphical User Interface (Figure 8.12). The interface allows users to navigate quickly through the video footage, showing parts that may contain a defect, and thus saving significant time and effort that are typically needed for evaluating the entire video file. Users can also override the system-suggested defects and their severity, or add comments. A final report that includes users' changes can then be created.

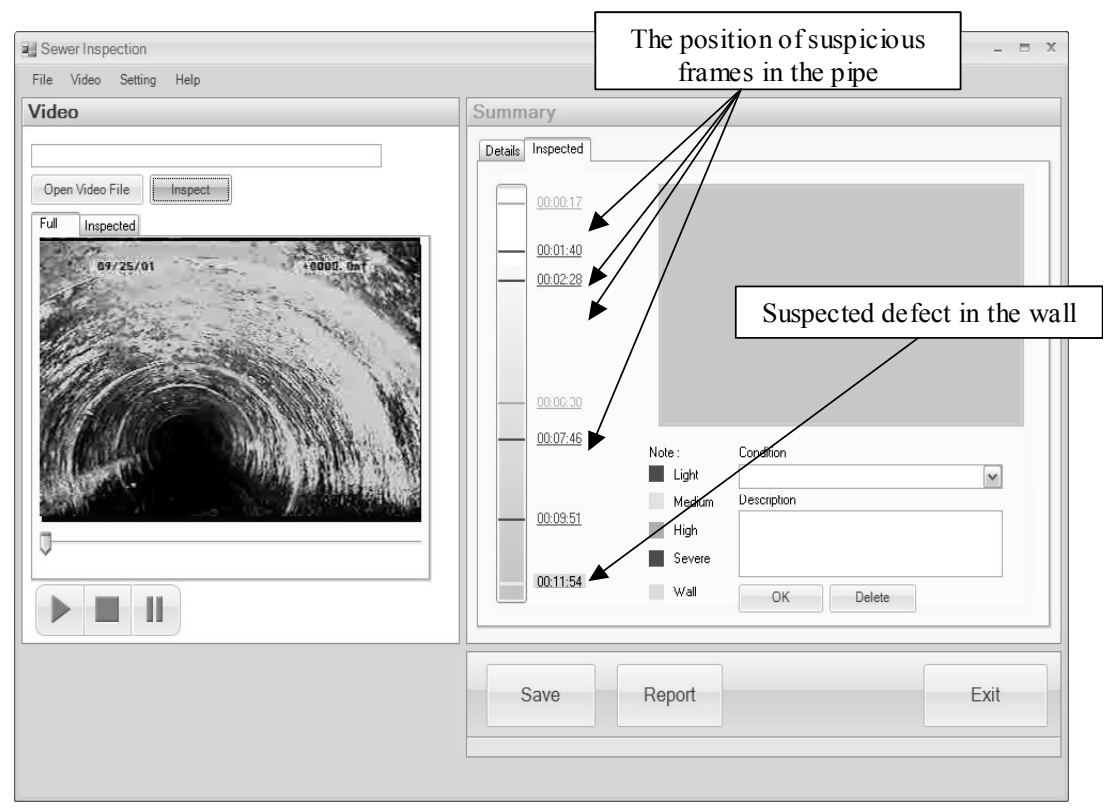

Figure 8.12 Interface for editing IIR. The user can quickly re-inspect the sewer. By clicking on each suspicious frame the video starts from that frame position. The user can also change the type and severity of the defects suggested by the system, if required. 


\subsection{Conclusions}

This chapter presented a novel approach and proof-of-concept software prototype to facilitate the storage, analysis, and management of sewer inspection data, and the semi-automatic extraction of historical condition data information from archived sewer inspection CCTV files. The software was successfully used to efficiently access, analyze, and evaluate sewer condition data from CCTV video files obtained from the City of Regina.The development and evaluation of the prototype software have proved very promising.

Further extension of the software will focus on rendering a more operational solution to the problem. The algorithms will need to be extended to address the current limitations exhibited by the software. Some of these limitations include the automatic detection of cracks, fractures, or deformation of the sewer, as well as estimating the severity of these defects. Extending the software to be used by CCTV inspection operators (i.e. using live video feeds instead of archived recordings) will also be another possible extension. Techniques for guiding the CCTV operator during the inspection process will also be investigated.

\section{Acknowledgment}

This work is a collaborative effort between the National Research Council, the University of Regina, and the City of Regina. The writers acknowledge financial support for this project from the Communities of Tomorrow, Inc. The authors thank Loretta Gette at the City of Regina for providing data and guidance throughout this project. The writers thank Dr. David Hubble and the anonymous reviewers for their valuable suggestions and feedback.

\section{References}

Gooch, R.M., Clarke, T.A., and Ellis, T.J. (1996). A semi-autonomous sewer surveillance and inspection vehicle. Proc. of IEEE Intelligent Vehicles Symposium, pp. 64-69, Tokyo, Japan, Sept. 19-20, 1996

Halfawy, M.R. (2008) "Integration of municipal infrastructure asset management processes: challenges and solutions,"ASCE Journal of Computing in Civil Engineering, 22 (3), pp. 216-229.

Halfawy, M.R., Dridi, L., Baker, S. (2008). "Integrated decision support system for optimal renewal planning of sewer networks," Journal of Computing in Civil Engineering, 22 (6), to appear (November 2008). 
Halfawy, M. R. and Figueroa, R. (2006). Developing enterprise GIS-based data repositories for municipal infrastructure asset management, Proc., Joint International Conference on Computing and Decision Making in Civil and Building Engineering, ICCCBE/ASCE/DMUCE/CIB, Montreal, Canada.

Halfawy, M.R., Pyzoha, D., and El-Hosseiny, T., (2002). An integrated framework for GIS-Based civil infrastructure management systems, Proceedings of the Canadian Society for Civil Engineers (CSCE) Conference, Montreal, Canada.

Halfawy, M.R., Pyzoha D., Young R., Abdel-Latif M., Miller R., Windham L. and Wiegand R. (2000) GIS-based sanitary sewer evaluation survey, 20th Annual ESRI International User Conference, Jun., San Diego, CA.

Iyer, S. and Sinha, S. (2005). Automated condition assessment of buried sewer pipes based on digital imaging techniques. J. Indian Inst. Sci., 85, 235-252.

Iyer, S. and Sinha, S. (2006). Segmentation of pipe images for crack detection in buried sewers. J. Computer-Aided Civil and Infrastructure Engineering, 21 (2006) 395-410.

Sinha, S. and Knight, M. (2004). Intelligent system for condition monitoring of underground pipelines. J. Computer-Aided Civil and Infrastructure Eng., 19 (1), 42-53.

Viola, P. and Jones, M. (2001). Rapid Object Detection using a Boosted Cascade of Simple Features. Proc. of IEEE Computer Society Conference on Computer Vision and Pattern Recognition, Vol. 1, 511-518.

WRc. (2001). Sewerage Rehabilitation Manual, Fourth Edition, Volumes I \& II, Water Research Centre, UK. 
